\title{
Bericht über die Gründung und erste Jahresversammlung der palaeontologischen Gesellschaft
}

\author{
von Herrn 0tto Juekel
}

Den Boden für die Begründung und Wirksamkeit einer Gesellschaft, die sich der Förderung der Palaeontologie und der Vertretung ihrer Interessen ausschließlich widmen sollte, glaubte ich dadurch ebnen zu müssen, daß ich fürs erste eine periodische Zeitschrift für unser Fach gründete. Ich möchte es an dieser Stelle dankbar anerkennen, daß Herr Dr. Thost, als Vertreter der Firma Gebrüder Borntraeger, kein Bedenken trug, einen diesbezüglichen Vertrag mit mir abzuschließen, trotzdem die Aussichten für die Rentabilität eines solchen Organs zunächst keineswegs günstig erschienen. Nachdem aber diese Grundlage für ein weiteres Vorgehen gewonnen war, versandte ich das nachstehende Rundschreiben.

Die Palaeontologie, die in Deutschland zuerst wissenschaftlich gepflegt wurde und in Männern wie Freiherr v. Schlotheim, Bronn, Goldfuß und später in K. v. Zittel Förderer gehabt hat, deren grundlegende Bedeutung weit über die Grenzen Deutschlands hinausreichte, ist in den letzten Dezennien in unserm Lande sehr vernachlässigt worden. Während andere Länder, namentlich Amerika, das Studium der Palaeontologie zu einem der populärsten Forschungs- und Lehrgebiete gemacht haben, fehlt dieser Wissenschaft in Deutschland noch jeder selbständige Stützpunkt'). Die praktische Verwertung der "Leitfossilien“ zar geologischen Altersbestimmung wird naturgemä $B$ der eigenen Bedentung der Fossilien als Organismen der Vorzeit, als Vorfahren der heutigen Tier- und Pflanzenwelt, vor allem aber ihrem dokumentarischen Wert für die wichtigsten Probleme der Entwicklungslehre, der vergleichenden

1) Gemeint waren hiermit selbständige Institute oder Museen, sowie Stellen für deren Leiter zur ausschlieblichen Förderung unseres Faches. 
Anatomie und der Morphologie in keiner Weise gerecht. Neben den geologisch-faunistischen Gesichtspunkten müssen diese morphologischen Momente der palaeontologischen Forschung stärker betont werden, es muß ihr eine selbständige Stellung zwischen der Geologie und der Zoologie bezw. Botanik eingeränmt werden. Nur wenn diese Nachbarwissenschaften direkt mit der Palaeontologie kommunizieren können, würde sie selbst die Freiheit haben, in ihren Vertretern sich nach ihren verschiedenen Richtungen ungehindert zu entfalten.

Zur Erreichung dieser Aufgaben bedarf es einer Gesellschaft, die die Vertretung der Interessen der Palaeontologie energisch in die Hand nimmt, sowie einer Zeitschrift, in der sie ihre Forschungsergebnisse, die bisher in den verschiedensten Nachbargebieten verstreut wurden, konzentrieren kann, und ihr gleichzeitig als Organ für ihre fachlichen Bestrebungen und für ihre Vertretung nach außen hin dienen würde.

Bei dem starken Rückgange, den die palaeontologische Forschung auch in der Zahl ihrer Vertreter in Deutschland erfahren hat, würde weder eine Gesellschaft noch eine Zeitschrift allein auf dem Boden Deutschlands auf einen großen Erfolg rechnen können. Ein solcher ist nur zu erreichen, wenn der Gesellschaft mindestens diejenigen Palaeontologen des Auslandes beitreten, die an der dentschen Literatur lebhaften Anteil nehmen und gelegentlich in dentscher Sprache ihre Arbeiten veröffentlichen. Es wäre aber zu hoffen, daß die zu begründende Gesellschaft die osteuropäischen Palaeontologen enger zusammenführte und auf dieser breiteren Grundlage eine internationale Bedeutung erlangte.

Die Vorbereitungen für die Begründung einer Palaeontologischen Zeitschrift hat der Unterzeichnete inzwischen zu vorläufigem Abschluß gebracht, für die Begründung einer Gesellschaft, deren Mitgliedern diese Zeitschrift für ihren Jahresbeitrag von $20 \mathrm{Mk}$. zustehen würde, sind ihm vou vielen Seiten aus dem In- und Auslande ernutigende Zuschriften zugegangen. An Euer Hochwohlgeboren ergeht nun die ergebene Anfrage, ob Sie der zu begründenden "Palaeontologischen Gesellschaft" beizutreten geneigt sind. Es ist geplant und mit dem Vorstande der Deutschen Geologischen Gesellschaft verabredet worden, die Begrïndung der neuen Gesellschaft bei Gelegenheit der diesjährigen Geologen-Versammlung in Greifswald vorzunehmen. Der Onterzeichnete beabsichtigt ferner, der dann begriindeten Gesellschaft den Vorschlag zn unterbreiten, ihre 
erste Jahresversammlung Anfang September in Halberstadt abzuhalten. Dieser Treffpunkt wäre besonders günstig im Herzen Deutschlands, am Fuße des Harzes gelegen, er würde den Mitgliedern außer den Anregungen, die diese sich gegenseitig bieten, Gelegenheit geben, die großartige Fundstätte der Dinosaurier und anderer Tierformen der Halberstädter Trias eingehend zu besichtigen, und sie würden in der alten Stadt, die auch ein palaeontologisch beachtenswertes Museum besitzt, freundlichster Aufnahme sicher sein.

Für gütige Mitteilung auf beiliegender Karte würde $z u$ besonderem Danke verpflichtet sein

Prof. Dr. Otto Jaekel.

Greifswald, im Mai 1912.

Auf dieses Rundschreiben hin meldeten bereits 136 Vertreter und Freunde der Palaenntologie ihren Beitritt an und erklärten sich mit dem Vorschlage einverstanden, eine konstituierende Versammlung in Greifswald bei Gelegenheit der dort vom 8. bis 10. August 1912 tagenden Jahresversammlung der Deutschen Geologischen Gesellschaft abzuhalten. Daselbst traten noch einige andere Mitglieder der Gesellschaft bei, so daß die Zahl der Anmeldungen nunmehr 143 betrug. Von diesen waren in Greifswald bei der Begründung der Gesellschaft am 12. August 1912 folgende Herren anwesend:

1. R. Bärtling, Dr., Berlin

2. F. Briest, Gutsoesitzer, Boltenhagen b. Grimmen

3. S. v. Bubnoff, Dr., Freiburg i. Br.

4. A. Dannenberg, Dr. Prof., Aachen

5. W. Deecke, Dr. Prof., Freiburg i. Br.

6. H. Fischer, Dr., Königsberg

7. F. Frech, Dr. Prof., Breslau

8. P. A. Grïssner, Oberbergrat, Schlachtensee b. Berlin

9. Höpke, cand. med., Greifswald

10. Freiherr F.v. Huene, Prof.Dr.,Tübingen

11. O. Jaekel, Prof. Dr., Greifswald

12. E. Kalkowsky; Dr. Prof. Geh. Hofrat, Dreşden

13. J. Kraenker, Dr. Prof., StraBburg i. E.

14. P. G. Krause, Dr. Prof., Eberswalde

15. E. Krenkel, Dr., Leipzig

16. R. Lachmann, Dr., Breslau
17. Leick, Oberlehrer Dr., Greifswald

18. F. Liebrecht, Dr., Lippstadt i. W.

19. V. Madsen, Dr. Direktor, Kopenhagen

20. H. Menze1, Dr., Berlin

21. H. Philipp, Dr., Greifswald

22. J. Pompeckj, Dr. Prof., Göttingen

23. H. Rassmass, Dr., Berlin

24. H. Rauff, Dr. Prof., Berlin

25. C. Renz, Dr., Breslau

26. Axel Schmidt, Dr., Stuttgart

27. W. Soergel, Dr., Freiburg i. Br.

28. R. Thost, Dr., Grob-Lichterfelde

29. A. Tornquist, Dr. Prof., Königsberg

30. E. Wepfer, Dr., Freiburg i. Br.

31. R. Wilckens, Dr., Greifswald

32. E. Wüst, Dr. Prof., Kiel

33. J. Wy sogorski, Dr. Custos, Hamburg

34. E. Zimmermann, Dr. Prof., Berlin 
In längerer Aussprache über die Ziele und Aufgaben der Gesellschaft wurde Einstimmigkeit darüber erzielt, daß die Gesellschaft alle Richtungen der Palaeontologie fördern und unter steter Aufrechterhaltung ihrer Beziehungen zur historischen Geologie die Verbindungen nit den morphologischen und biologischen Wissenschaften pflegen wolle. Diesen Aufgaben sollen allgemeine Jahresversammlungen und das Organ, die "Palaeontologische Zeitschrift", dienen, die im Verlage von Gebrüder Borntraeger von Herrn Jaekel herausgegeben werden sollte. Man einigte sich ferner darüher, daß ein Vorstand und ein Beirat mit ähnlichen Funktionen, wie in der Geologischen Gesellschaft, die Geschäfte leiten solle, und daß die formelle Geschäftsführung im wesentlichen von der Verlagsbuchhandlung Gebrüder Borntraeger übernommen werde. Es erschien allen Mitgliedern unzweckmäßig, daß eine Bibliothek oder wissenschaftliche Sammlungen angelegt würden, wohl aber solle die Gesellschaft dahin zu wirken suchen, daB die Hilfsmittel unserer wissenschaftlichen Tätigkeit von dem Staate in stärkerem Maße als bisher gefördert werden möchten.

Zum provisorischen Vorstand für ein Jahr bezw. bis zur nächstjährigen Hauptversammlung wurden die Herren Jaekel, Pompeckj und Frech gewählt. Als Ort der vorjährigen ersten Versammlung wurde Halberstadt gewählt, wo Herr Jaekel seine neuesten Funde von Dinosauriern und anderen Trias-Wirbeltieren demonstrieren wollte.

Diese erste allgemeine Versammlung tagte am 5. und 6. September in Halberstadt im Hotel "Weißes Roß", wobei Herr Jaekel die Leitung der Geschäfte übernahm. Nachdem er in der ersten Sitzung am 5. September, vormittags $9 \mathrm{Uhr}$, einen Überblick über die bisherigen Maßnahmen zur Begründung der Gesellschaft gegeben, und die Ziele derselben nochmals kurz erläutert hatte, hielt er einen Vortrag über die Wege und A ufgaben der Palaeontologie. Der Vortragende erblickt die Hauptaufgabe der neuen Gesellschaft darin, daB sie die Interessen der Palaeontologie in jeder Hinsicht vertritt und ihr vor allem Bewegungsfreiheit im Rahmen der naturwissenschaftlichen Fächer verschafft. Sie solle nicht nur die geologische, sondern vor allem die morphologisch-biologische Seite der Palaenntologie pflegen und dahin wirken, daB der Nachwuchs unseres Faches nicht mehr wie bisher genötigt ist, seine Ausbildung in erster Linie auf mineralogisch-geologischer Unterlage zu suchen, sondern das Studium der zur Beurteilung fossiler Organismen unerläßlichen Kenntnisse der lebenden und ihrer Lebensverhältnisse in den Vordergrund stellen kann, die Palaeontologen also in die 
Lage versetzt werden, ihre hauptsächlichste Aushildung nicht mehr auf chemisch-physikalischer, sondern auf biontologischer Grundlage zu suchen. Die eigenartige historische Stellung der Palaeontologie würde dabei selbstverständlich eine gründlichste geologische Vorbildung niemals entbehren können und es sei auch nicht zu befürchten, daß in unserem Fache die geologisch-faunistische Seite zu kurz kommen würde, da sicherlich nach wie vor ein großer Teil der Palneontologen gleichzeitig Geologen sein und von dieser Seite her unsere Wissenschaft fördern würden. Dieser Vortrag, an dessen Besprechung sich namentlich die Herren Pompeckj und Fraas beteiligten, sollte als Aufsatz in den ersten Heft unserer Zeitschrift zum Abdruck gelangen.

In ẗbrigen wurden in dieser Sitzung zalhlreiche Fragen der inneren Einrichtung und äußeren Gestaltung unserer Gesellschaft besprochen, eine Beschlußfassung hierüber für die Sitzung am folgenden Tage vorgesehen.

Am 5. September nachmittags begaben sich die Teilnehmer an der Versammlung in die Tongrube der Baereckeschen Ziegelei, wo Herr Jaekel zunächst die geologischen Verhältnisse des Fundortes und die Gliederung der Keuperschichten am nördlichen Harzrande besprach und dann auf die Wirbeltierfunde näher einging. Im ganzen waren seit 1909 etwa 35 mebr oder minder vollständige Skelette von Dinosauriern, Keste mehrerer Schildkröten, ein Miosaurier, Belodonten und zahlreiche Reste verschiedener Fischformen gefunden, unter denen ein Zahn von Ceratodus polymorphus Miall die oberen sandigen Schichten der Tongrube in das Rät zu verweisen scheint. Die darunter gelegenen Tone, die die zusammenhängenden Dinosaurierreste enthalten, gehören jedenfalls dem mittleren Keuper an. Das erste Heft unserer Zeitschrift soll eine Übersicht über die bisherigen Funde bringen. Den Besuchern konnte in der Grube ein heranspräpariertes Skelett eines Plateosauriden (XXXVI) gezeigt werden, das die meisten Knochen des Rumpfes und Schwanzes, aber nur wenige Reste des Halses und Kopfes enthält. In situ konnte das Skelett XLI beobachtet werden, wo gerade ein Stück des Schwanzes nit 22 Wirbeln, zahlreiche Wirbel, Rippen und Fußknochen freigelegt waren. Auch ein vorderes Rumpfstück mit beiden Armen XLIII war im Zusammenhang der Teile aufgedeckt. Besonders bewundert wurde ein nahezu vollständiger Panzer einer Schildkröte, die der Proganochelys Quenstedti Baur aus Württemberg nahesteht und schon damals auch einige Knochen des inneren Skeletts, so namentlich den wichtigen Schulterbogen erkennen ließ. 
Im Anschluß an den Besuch der Baereckeschen Tongrube wanderten die Teilnehmer über die Klussberge und Thekenberge zu dem dort neu angelegten Flugplatz, wo die nenesten Flugapparate eingehend besichtigt werden konnten, nach Spiegelsbergen, wo der Versammlung seitens der Stadt ein Ehrentrunk gereicht wurde.

Am 6. September fand vormittags zunächst eine Geschäftssitzung statt, um vor allen den in Greifswald gewählten provisorischen Vorstand durch einige Nachwahlen zu ergänzen. Derselbe setzt sich nunmehr wie folgt zusammen:

\section{Präsident Herr Jaekel}

Vizepräsident die Herren Frech und Pompeckj

Schriftführer die Herren v. Arthaber und Frbr. v. Huene

Schatzmeister Herr P. G. Krause

Als Mitglieder des Beirates wurden gewählt die Herren Fraas-Stuttgart, Rauff-Berlin, Smith-Woodward-London, Schuchert-New Haven, Conn., Tschernyschew-St.-Petersburg, Wiman-Upsala.

In eine Kommission zur Beratung der Statuten wurden gewählt die Herren Fraas, Frech, Jaekel, Pompeckj und Thost. Dieselbe soll der nächsten Jahresversanmlung einen ausgearbeiteten Statutenentwurf vorlegen und folgende Direktiven beachten:

Die Gesellschaft wünscht nur persönliche Mitglieder zu ernennen, zu deren Aufnahme der Vorschlag dreier Mitglieder und die Verkündung durch den Vorstand genügt. Das Geschäftsjahr soll mit dem Kalenderjahr zusammenfallen. Der Beitrag von $20 \mathrm{M}$. soll erst vom nächsten Jahre $a b$ erhoben werden ${ }^{1}$ ), jedes Jahr an 1. Jannar fällig sein und am 1. April eingezogen werden können. Der Vorstand soll in seinen einzelnen Stellen etwa alle drei Jahre wechseln, ebenso der Beirat. Anszunehmen sind von diesem Zwange die Stellen des Schatzmeisters und tes Redakteurs. Die Zeitschrift soll außer den Originalanfsätzen Berichte über wichtige Vorgänge und Publikationen im Fache, Sammelreferate iuch über Fortschritte in den morphologischen Nachbarfïchern bringen. Die Gesellschaft soll die Vertretung unseres Faches in allgemeinen Fragen wie anch in einzelnen Vorgängen in die Hand nehmen und sich über die Regelung solcher Probleme der Nomenklatur, Terminologie und Systematik gegebenenfalls mit den Nachbarwissenschaften in Verlindung setzen.

1) Die bereits eingezahlten Beiträge werden auf das nächste Jahr übertragen. 
Die Mitglieder unterstützen den Vorstand besonders auch darin, daB sie eingesandte Manuskripte auf Wunsch einer kritischen Durchsicht unterziehen.

Auf den Antrag des Herrn Pompeckj, möglichst bald eine volle Fusion der Zeitschrift mit der Gesellschaft herbeizuführen, erklärte sich Herr Jaekel bereit, seine Rechte und Pflichten in dem Verlagsvertrage der Zeitschrift an die Gesellschaft zu übertragen, hielt es jedoch für wünschenswert, die Leitung der Zeitschrift einige Jahre zu behalten. Auf diesbezügliche Zusicherungen der Versammlung übertrug Herr Jaekel seinen Verlagsvertrag mit der Firma Borntraeger auf die Gesellschaft. Die Firma Borntraeger übernahm es daraufhin und mit Rücksicht auf das schnelle Anwachsen der Mitgliederzahl, den Vertrag in einem für die Gesellschaft günstigen Sinne umzugestalten. Der Unfang des Bandes, der für die illustrative Ausstattung desselben vorgesehene Betrag soll anf 1000 Mark erhöht und den Autoren 50 Separata gratis geliefert werden.

Auf Antrag der Herren Pompeckj und P. G. Krause beschloß die Versammlung, daß die Palaeontologische Gesellschaft für eine Neuauflage von Zittels Handbuch wirken möge, und ernannte zur Förderung dieses Planes eine Kommission aus den Herren Pompeckj, Jaekel und Krause.

Herr Jaekel wurde auf seinen Antrag beauftragt, Schritte zu tun, daß sich die Palaeontologische Gesellschaft dem Vorgehen der Dentschen Zoologischen Gesellschaft in sachgemäßer Weise anschließe, auf eine Einschränkung der absoluten Gültigkeit des Prioritätsgesetzes hinzuwirken. Es wird hierbei nach Möglichkeit darauf zu achten sein, daß den Beschlüssen der zusammengetretenen palaeontologisch-nomenklatorischen Kommission auf dem letzten internationalen Geologenkongresse in Stockholm, deren Präsident Herr Frech ist, tunlichste Geltung verschafft wird.

In dem wissenschaftlichen Teil dieser Sitzung sprach Herr WimanUpsala: Über die palaeontologische Bedeutung des Massentodes unter den Tieren, Herr Fraas-Stuttgart: Über die ältesten Schildkröten im Keuper Württembergs und Herr Semper-Aachen: Über einige philosophische Probleme in der geologischen und palaeontologischen Forschung.

Über den Ort der nächsten Tagung wurde zunächst ein definitiver Beschluß noch nicht gefaßt. Herr Fraas bat, einen Besuch in Stuttgart noch um zwei bis drei Jahre hinauszuschieben, da er bis dahin noch mit der Erweiterung seines Museums beschäftigt sein dürfte. Inzwischen ist von Herrn Kalkowsky-Dresden eine Einladung an die Gesellschaft ergangen, als nächsten Versammlungsort Dresden zu wählen. 
Der Zeitpunkt soll noch vorbehalten bleiben, wird aber voraussichtlich auf den Anfang Oktober verlegt werden, um Kollisionen mit anderen Versammlungen zu vermeiden. In dankenswerter Weise hat sich auch Herr Dr. Dollo bereit erklärt, später die Versanmlung nach Brüssel einzuladen.

Den Abschluß fand diese zweite Sitzung ihrer ersten allgemeinen Tagung mit dem einstimmigen Beschluß, den hervorragenden Verdiensten, die sich die Herren Ch. E. Walcott, Henry F. Osborn und Louis Dollo um die Förderung der Palaeontologie erworben haben, dadurch anzuerkennen, daß sie die genannten Herren zu ihren Ehrenmitgliedern ernennt.

Von der in Halberstadt gewählten Kommission zur Vorbereitung der Satzungen unserer Gesellschaft ist inzwischen folgender Statutenentwurf ausgearbeitet worden:

\section{Satzungen der Palaeontologischen Gesellschaft}

(Entwurf)

\section{§ 1. Name, Zweck und Sitz der Gesellschaft}

Die am 8. August 1912 in Greifswald gegründete wissenschaftliche Vereinigung führt den Namen Palaeontologische Gesellschaft. Sie hat den Zweck, eine Vereinigung von Palaeontologen des In- und Auslandes zu bilden, um die Interessen der Palaeontologie nach allen Richtungen zu fördern und zu vertreten. Der Sitz der Gesellschaft ist Berlin.

\section{$\S 2$.}

Die Palaeontologische Gesellschaft sucht ihren Zweck zu erreichen

1. durch Jahresversammlungen, die an einem Orte des In- oder Auslandes stattfinden,

2. durch Herausgabe von Veröffentlichungen, vor allem der periodisch erscheinenden Zeitschrift,

3. durch Beteiligung an anderweitigen wissenschaftlichen Veranstaltungen, von denen eine Förderung palaeontologischer Interessen zu erwarten ist.

$\$ 3$.

Als "Versammlungen der Mitglieder" im Sinne der $\S \S 32 \mathrm{ff}$. des $\mathrm{BGB}$. gelten nur die Jahresversammlungen, die vorher allen Mitgliedern angekündigt sind. 


\section{$\S 4$. Mitglieder betreffend}

Mitglied der Palaeontologischen Gesellschaft kann jeder werden, welcher die Zwecke derselben zu fördern gewillt ist und hierzu seiner Lebensstellung oder wissenschaftlichen Ausbildung nach für befähigt zu erachten ist.

$\S 5$.

Die Aufnahme in die Gesellschaft erfolgt auf schriftliche oder anch mündliche Meldung innerhalb einer Versammlung durch zwei Mitglieder bei dem Präsidenten, der die Bekanntmachung sofort vornehmen oder den Antrag an den Vorstand verweisen kann. Die Namen der neu aufgenommenen Mitglieder werden in der Zeitschrift veröffentlicht.

\section{$\S 6$.}

Die Mitglieder zahlen einen Jahresbeitrag von 20 M., welcher am 1. Januar fällig ist und vom 1. April ab durch die Geschäftsführung durch Postnachnahme eingezogen werden kann. Im Laufe des Jahres eintretende Mitglieder zahlen bei der Aufnahme den vollen Jahresbetrag, außer im letzten Quartal, wenn sie ausdrücklich auf die Zustellung des laufenden Jahrgangs der Zeitschrift verzichten. Das Vereinsjahr beginnt mit dem 1. Januar.

\section{$\S 7$.}

Allen Mitgliedern gehen die Hefte der Palaeontologischen Zeitschrift durch deren Verlagsanstalt unentgeltlich $\mathrm{zu}$, vorausgesetzt, daB sie ihren Jahresbeitrag entrichtet und die Verlagsanstalt rechtzeitig von Änderungen ihrer Adresse in Kenntnis gesetzt haben.

$\S 8$.

Jedes Mitglied hat Anspruch auf die Ankündigung der Jahresversammlnng und aller anderen allgemeinen Veranstaltungen der Gesellschaft, insonderheit auf die Zustellung von Wahlkarten für die Wahl des Vorstandes und des Ortes der nächsten Jahresversammlung. Diese Wahlkarten müssen mit namentlicher Unterschrift an den Präsidenten übersandt werden. Dieser ist nicht berechtigt, die Namensunterschriften bei der Verlesung der Stimmen bekannt zu geben.

\section{$\$ 9$.}

Jedes durch Spezialarbeiten bekannt gewordene Mitglied ist verpflichtet, auf den ausdrücklichen Wunseh des Prüsidenten oder des Herausgebers der Zeitschrift ein Urteil über eine in sein Spezialgebiet fallende, zur Veröffentlichung eingesandte Arbeit abzugeben, falls ïber deren Aufnahme Meinungsverschiedenheiten bestehen. 
$\S 10$.

Die Mitgliedschaft hört auf:

1. durch freiwilliges Ausscheiden, das dem Vorsitzenden vor Beginn des neuen Geschäftsjahres schriftlich angezeigt ist,

2. durch den Tod des betreffenden Mitgliedes,

3. durch Ausschluß, wenn das betreffende Mitglied den Jahresbeitrag zu zahlen verweigert oder sonstige Gründe den Vorstand dazu veranlassen. Im letzteren Falle steht dem Betreffenden das Berufungsrecht an die Jahresversammlung zu, die alsdann über den Ausschluß mit zwei Drittel Mehrheit entscheidet.

\section{$\S 11$.}

Die Gesellschaft kann Ehrenmitglieder ernennen, die sich um die Palaeontologie oder die Gesellschaft besondere Verdienste erworben haben. Ihre Ernennung erfolgt entweder auf einstimmigen Beschluß des Vorstandes, oder auf Vorschlag des Vorstandes durch die allgemeine Versammlung mit zwèi Drittel Mehrheit. Die Ehrenmitglieder gehören zu den Mitgliedern der Gesellschaft, haben deren Rechte, zahlen jedoch keinen Beitrag.

\section{$\S 12$.}

Die Gesellschaft kann in ihrer Jahresversammlung auf Vorschlag des Vorstandes oder Beirates korrespondierende Mitglieder ernennen, die von Nachbargebieten der Palaeontologie aus zu deren Förderung beigetragen haben. Sie gehören nicht zu den Mitgliedern der Gesellschaft im engeren Sinne und haben auch keinen Anspruch auf kostenlose Znstellung ihrer Veröffentlichungen.

\section{$\S 13$. Geschäftsführung}

Die Geschäftsführung wird durch einen Vorstand und einen Beirat besorgt. Die Wahl ihrer Mitglieder erfolgt durch die Jahresversammlung nach absoluter Mehrheit der abgegebenen Stimmen, wobei die von answärts eingegangenen Stimmen bei jedem Wahlgange mitzuzählen sind. Zur Vorbereitung der Wahl ist der Vorstand verpflichtet, jedes Mitglied vier Wochen vor der Wahl über die bisherige Zusammensetzung der Geschäftsführung und die sich daran knüpfende Möglichkeit einzelner Wiederwahlen zu unterrichten, auch Vorschläge des Beirates für Nellwahlen des Vorstandes zur Kenntnis zu bringen. Falls eine Jahresversammlung ausfällt, verlängert sich die Amtsdauer der Geschäftsführung bis zur nächstfolgenden Jahresversammlung. Jedes Mitglied des Vor- 
standes und Beirates, mit Ausnahme des Herausgebers der Zeitschrift, wird für ein Geschäftsjahr gewählt. Eine zweimalige Nachwahl ist zulässig. Eine Ersatzwahl für ausscheidende Mitglieder des Vorstandes oder Beirates kann durch Kooptation dieser Körperschaften erfolgen.

\title{
$\S$ 14. Der Vorstand
}

Der Vorstand besteht aus

\author{
einem Präsidenten, \\ zwei Vizepräsidenten, \\ zwei Schriftführern, \\ einem Schatzmeister; \\ dem Herausgeber der Zeitschrift.
}

Der Vorstand ist lediglich gewählt zur Erreichung der Zwecke der Gesellschaft, nicht im Sinne der $\$ \S 26$ ff. des BGB. Vorstand im Sinne der $\$ \S 26$ ff. des BGB. ist der jeweilige Präsident.

Der Präsident leitet die Versammlungen sowie die Wahlen und bestimmt die Tagesordnung der Versammlungen. Er verwaltet das Archiv der Gesellschaft. Seine Vertretung erfolgt durch die Vizepräsidenten nach Maßgabe ihres Wahlalters. Die Schriftführer verfassen die Protokolle und Berichte über die Verhandlungen und Veranstaltungen, und erledigen den Schriftwechsel, soweit dies dem Präsidenten zweckmäßig erscheint. Der Schatzmeister besorgt die Verwaltung des Gesellschaftsvermögens und überwacht die Einziehung der Beiträge und die Geschäfte der Gesellschaft, die von der Verlagsanstalt der Palaeontologischen Zeitschrift übernommen sind. Die Prüfung seiner Verwaltung erfolgt in der Jahresversammlung durch die Revisoren, die der Versammlung spätestens am letzten Sitzungstage darüber Bericht zu erstatten haben.

Der Herausgeber der Zeitschrift leitet diese im Auftrage der Gesellschaft nach Maßgabe ihrer Satzungen und des Vertrages mit der Verlagsanstalt. Er ist dem Vorstande und der Gesellschaft hierfür verantwortlich. Er hat Material für die Zeitschrift zu sanmeln und kann über die Aufnahme oder Ablehnung eingesandter Arbeiten vorläufig entscheiden. Gegen seine Entscheidung ist eine Berufung in erster Instanz an den Beirat, in letzter Instanz an die Jahresversammlung zulässig. Es steht ihm das Recht zu, eingehende Arbeiten an Mitglieder der Gesellschaft zum Referat zu übersenden, und im Falle von Meinungsverschiedenheiten mit dem Referenten die Pflicht, das Präsidium um eine Entscheidung anzugehen. 


\section{$\S$ 15. Beirat}

Der Beirat besteht aus sechs Mitgliedern, die in der Jahresversammlung auf die Daner von drei Jahren gewählt werden und nach Ablauf dieser Zeit für den gleichen Zeitraum nicht wieder wählbar sind. Jedes Jahr scheiden zwei Mitglieder aus. Bei der Zusammensetzung des Beirates sollen die ausländischen Mitglieder tunlichst berücksichtigt werden. Der Beirat hat die Aufgabe, die wissenschaftlichen Leistungen der Gesellschaft und die Förderung ihrer Interessen durch den Vorstand zu überwachen und das Recht, diesbezügliche Rechenschaft von dem Vorstande zu fordern. Den Mitgliedern des Beirates werden vom Vorstande der Gesellschaft alle etwaigen Anträge auf Satzungsänderungen unterbreitet. Der Beirat bildet bei Streitigkeiten zwischen dem Vorstande und den Mitgliedern eine Berufungsinstanz, gegen deren Entscheidung nur noch diejenige der Hauptversammlung angerufen werden kann.

Den Vorsitz im Beirat führt der nach dem Alphabet ältere der beiden Beiräte, die dieser Körperschaft am längsten angehören, der andere derselben hat gegebenenfalls seine Vertretung zu übernehmen.

Bei den Jahresversammlungen findet eine gemeinsame Sitzung vom Vorstand und Beirat unter dem Vorsitz des Präsidenten der Gesellschaft statt.

Bei allen Abstimmungen des Beirates zählen auch schriftliche Meinungsäußerungen seiner Mitglieder.

\section{\$ 16. Jahresversammlung}

Die Jahresversammlung soll in erster Linie wissenschaftlichen Zwecken dienen und den Mitgliedern tunlichst nicht nur Gelegenheit zu gegenseitiger Anregung und Förderung, sondern auch zur Besichtigung wichtiger palaeontologischer Sammlungen oder Fundorte bieten. Sie nimmt die Wahl des Vorstandes und Beirates vor, prüft deren Geschäftsfïhrung und nimmt den Bericht der zwei für das Geschäftsjahr gewählten Revisoren entgegen. Sie entscheidet über Anträge zur Änderung der Satzungen nur dann sofort, wenn solche von dem Vorstande und Beirat zugleich beantragt werden, als eilig genügend motiviert und jedem Mitgliede vier Wochen vorher angekündigt sind, andernfalls erst in der nächsten Jahresversammlung. Ort und Zeit der Jahresversammlung sind nicht begrenzt, müssen aber vom Vorstande den Mitgliedern mindestens drei Monate vorher angemeldet werden. 


\section{$\$ 17$. Abstimmungen}

Abstimmungen erfolgen im allgemeinen durch einfache Mehrheit. $\mathrm{Zu}$ Satzungsänderungen und Ausschluß von Mitgliedern sind zwei Drittel der gültigen Stimmen erforderlich, bei Wahlen zu Ehrenmitgliedern drei Viertel.

\section{$\$ 18$. Auflösung der Gesellschaft}

Über die Auflösung der Gesellschaft kann nur in einer allgemeinen Versammlung (Jahresversammlung) entschieden werden. $\mathrm{Zu}$ einem solchen Beschluß sind zwei Drittel der Stimmen sämtlicher abstimmenden Mitglieder erforderlich. Die die Auflösung bestimmende Versammlung hat gleichzeitig über die Verwendung des Gesellschaftsvermögens Bestimmungen $\mathrm{zu}$ treffen.

Dieser Entwurf soll der nächsten Jahresversammlung zur Beschlußfassung vorgelegt werden. Es ist sehr wünschenswert, daß Vorschläge auf Abänderung dieses Entwurfes dem Präsidium möglichst noch vor der nächsten Jahresversammlung zugestellt werden.

Die nächste Jahresversammlung wird gemäß einer sehr dankenswerten Zusage des Herrn Kalkowsky in Dresden stattfinden, und zwar am 25. bis 27. September. Ein näheres Programm für diese Tagung wird den Mitgliedern zugehen. Es ist zu hoffen, daß von seiten unserer Mitglieder Vorträge und Demonstrationen für diese Tagung vorbereitet und bei dem Präsidium rechtzeitig angemeldet werden. Wie aus unserem Statutenentwurfe hervorgeht, möchten wir die Jahresversammlungen keineswegs auf die Grenzen Deutschlands beschränken, sondern dabei wichtige Stützpunkte unseres Faches auch im Auslande anfsuchen. Es ist sehr dankenswert, daß sich unser Ehrenmitglied Herr L. Dollo bereit erklärt hat, die Gesellschaft in einem der nächsten Jahre in Brüssel za empfangen. Auch Herr Eb. Fraas hat uns eine solche Einladung nach Stuttgart in Aussicht gestellt.

Bisher sind der Gesellschaft folgende Mitglieder beigetreten. Argentinien.

Albert Bracht, Dr. R. Stappenbeck, Dr. A. Windhausen. Australien.

Ludwig Glauert. Belgien.

Prof. Dr. Louis Dollo, Prof. H. de Dorlodot. 
Bulgarien.

Dr. Stephan Bontschew.

Dänemark.

Dr. Victor Madsen, Dr. med. Malling, Dr. J. P. J. Ravn, Frl. A. Hintze, Herbert Winge.

Deutschland.

Dr. phil. K. Andree, Prof. Dr. L. von Ammon, Dr.Th. Arldt, P. Bamberg, Bärthold, Dr. Rich. Bärtling, Dr. Karl Beck, Prof. Dr. F. Beyschlag, Prof. Dr. J. Böhm, Wilhelm Bölsche, Prof. E. Bracht, F. Briest, Prof. Dr. Ferd. Broili, Dr. S. von Bubnoff, Dr. Hans Cloos, Prof. D. Dannenberg, Privatdoz. Dr. Daqué, Prof. Dr. Deecke, Prof. D. Deiser, Prof. Dr. Denckmann, Paul Dienst, Dr. Drevermann, Dr. G. Dyhrenfurth, Dr. Hans Fischer, H. Fromme, Prof. Dr. E. Fraas, Dr. Fliegel, Prof. Dr. J. Felix, Prof. Dr. Frech, Fremdling, Prof. Dr. Fricke, Dr. med. Froriep, M. Froriep, Heinr. Tauver von Gallenstein, C. von Goldbeck, Oberbergrat Graeßner, Prof. Pr. Gürich, Arthur von Gwinner, Dr. W. Haack, Dr.-Ing. Hambloch, Dr. phil. et med. Hamm, Bernhard Hauff, Bergrat M. Heckel, Hemprich, Dr. Bruno Hoffmann, Cand. med. Höpke, Dr. Freiherr Friedrich von Huene, Prof. Dr. Jaekel, Prof. Dr. Janensch, Dr. E. Jaworski, Carlo Joos, Prof. Dr. Kalkowsky, Prof. Dr. H. Klaatsch, Kurt Klamroth, Dr. F. Krantz, Dr. F. Klinghardt, Prof. Dr. von Koenen, Dr. phil. M. von Komorowicz, Dr. phil. Fr. Krantz, Prof. Dr. Krause, Dr. E. Krenkel, Dr. L. Krumbeck, Bergassessor Kukuk, Dr. R. Lachmann, Generalleutnant O. von Lauenstein, Dr. E. Leick, Dr. Otto von Linstow, Dr. F. Liebrecht, Dr. Ernst Meister, Dr. Hans Menzel, Dr. H. L. F. Meyer, Meyer-Libnitz, Prof. Dr. Michael, Dr. Aug. Müller, Dr. Hugo Mylius, Bergrat Neubauer, Prof. Dr. Konrad Oebbecke, Dr. Hans Philipp, Dr. Edm. Picard, Prof. Dr. Pohlig, Prof. Dr. Plieninger, Prof. Dr. Pompeckj, Prof. Dr. Herm. Rauff, Gustar Rebling, Dr. Hans Reck, Prof. Dr. Freiherr E. Stromer von Reichenbach, Prof. Dr. K. Reiser, Dr. Renz, Prof. Dr. A. Rothpletz, Dr. Hans Salfeld, Prof. Dr. W. Salomon, Dr. jur. P. Scherber, Stud. Heinr. Schliephake, Dr. Max Schlosser, Dr. Axel Schmidt, Prof. Dr. Martin Schmidt, Dr. A. Schrammen, Dr. H. Schroeder, Prof. Dr. F. E. Schulze, Prof. Dr. Max Semper, Dr. Wolfgang Soergel, Dr. Hans von Staff, Prof. Dr. G. Steinmann, Prof. Dr. Stolley, Hermann StrubeSchlanstedt, Dr. Benno Stürtz, Dr. Heinr. Taeger, Prof. Dr. Tornquist, Dr. Rob. Thost, Dr. J. Versluys, Prof. Dr. Joh. Walther, Dr. S. Wanner, Prof. Dr. Max Weber, Prof. Dr. H. Wedde, Dr. K. Wedekind, Dr. R. N. Wegener, Prof. Dr. Th. Wegener, Prof. E. Weise, Dr. W. 
Weisermel, Dr. E. Wepfer, Prof. Dr. Otto Wilkens, Dr. Rud. Wilckens, Dr. Wilh. Wunstorf, Prof. Dr. Ewald Wüst, Dr. Wysogorski, Konrad Zechlin, Prof. Dr. Ernst Zimmermann.

England.

Dr. F. A. Bather, H. N. Hutschinson, J. B. Kitschin, Dr. Arthur Smith Woodward.

Frankreich.

Gustave F. Dollfus, Prof. Dr. Charles Barrois. Griechenland.

Prof. Dr. Theod. Skouphos.

Italien.

Prof. Dr. Francesco Bassani.

Japan.

Prof. Dr. H. Yabe.

Java.

Dr. L. Rutten.

Mexiko.

Dr. C. Burckhardt.

Niederlande.

J. Eb. Bonnemann.

Norwegen.

A. E. Drolsum, Prof. Joh. Kjär. Österreich-Ungarn.

Prof. Dr. Lörenthey, Dr. Stefan von Gaal, Prof. Dr. Blaas, Dr. Karl

A. Redlich, Dr. Mauriz Remes, Prof. Dr. Philipp Počta, Prof. Dr. Franz Ryba, Prof. Dr. G. von Arthaber, Dr. Raimund Folgner, Dr. phil. Joseph Prosche, Dr. Oskar Ritter von Troll, Prof. Dr. Franz Toula, Baron Dr. phil. Franz Nopesa, Prof. Dr. Kramberger. Rumänien.

Prof. Dr. John Simionescu, Dipl.-Ing. Oskar Widenmeyer. RuBland.

Prof. Nic. Andrussow, Prof. Dr. N. Karakasch, Dr. A. Karpinsky,

Prof. A. P. Pavlow, Dimitry Sokolow, Dr. Tolmatschew, Theodor Tschernyschew.

Schweden.

Dr. A. Andersson, Prof. Dr. Moberg, Prof. Carl Wimann. Schweiz.

Dr. Bluntschli, Dr. Aug. Buxtorf, Prof. Dr. Hescheler, Dr. H. P. Stehlin. Serbien.

Prof. Zimbo J. Joczimowitsch. 
Süd-West-Afrika.

Dr. Lotz.

Transvaal.

Dr. E. C. N. van Hoepen M. I.

U. S. Amerika.

Rob. Anderson, Dr. Ralph Arnold, Edward W. Berry, Prof. E. Case, Prof. Dr. Clark, Prof. Dr. John Clarke, Prof. Bashford Dean, Prof. Dr. Eastman, Prof. J. B. Gilbert, Prof. Dr. Grabau, Henry F. Osborn, Roy L. Moodie, Prof. Dr. Sardeson, Prof. Charles Schuchert, Prof. W. Sinclair, Burnett Smith, Prof. Will. H. Twenhofel, Thomas Wayland Vaughan, Ch. D. Walcott, Stuart Weller, Prof. S. W. Williston, Chest. A. Reeds.

Wir bitten diese noch vielfach unvollständigen Adressen zu revidieren und zu vervollständigen, so daß wir am Schlusse des ersten Jahrganges eine definitive Übersicht über den Bestand unserer Gesellschaft bieten können. Wir bitten, ihr auch Freunde unseres Faches aus Laienkreisen zuführen $\mathrm{zu}$ wollen, die sich solchen wissenschaftlichen Gründungen gegenüber zunächst zurückzuhalten pflegen.

Alle Einsendungen für die Zeitschrift bitten wir an

Herrn Prof. Dr. Otto Jaekel in Greifswald, alle Geldsendungen direkt an die Verlagsfirma

Gebrïder Borntraeger, Berlin W. 3 כ5

richten $\mathrm{zu}$ wollen. 